Canadian

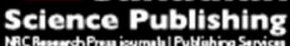

Applied Physiology, Nutrition, and Metabolism Physiologie appliquée, nutrition et métabolisme

\title{
Effect of participants' static stretching knowledge or deception on the responses to prolonged stretching
}

\begin{tabular}{|r|l|}
\hline Journal: & Applied Physiology, Nutrition, and Metabolism \\
\hline Manuscript ID & apnm-2016-0241.R1 \\
\hline Manuscript Type: & Article \\
\hline Complete List of Authors: & $\begin{array}{l}\text { Janes, W.C.; Memorial University of Newfoundland, School of Human } \\
\text { Kinetics and Recreation } \\
\text { Snow, Brandon; Memorial University of Newfoundland, School of Human } \\
\text { Kinetics and Recreation } \\
\text { Watkins, Caisie; Memorial University of Newfoundland, School of Human } \\
\text { Kinetics and Recreation } \\
\text { Noseworthy, Elecia; Memorial University of Newfoundland, School of } \\
\text { Human Kinetics and Recreation } \\
\text { Reid, Jonathan; Memorial University of Newfoundland, School of Human } \\
\text { Kinetics and Recreation } \\
\text { Behm, David; Memorial University of Newfoundland, }\end{array}$ \\
\hline Keyword: & \begin{tabular}{l} 
placebo, force, hamstrings, rate of force development \\
\hline
\end{tabular} \\
\hline \multicolumn{2}{|c}{} \\
\hline
\end{tabular}


Effect of participants' static stretching knowledge or deception on the responses to

$$
\text { prolonged stretching }
$$

\author{
AUTHORS: W.C. Ian Janes, Brandon B.G. Snow, Caisie E. Watkins, \\ Elecia A.L. Noseworthy, Jonathan C. Reid, David G. Behm \\ INSTITUTION: $\quad$ School of Human Kinetics and Recreation \\ Memorial University of Newfoundland \\ 230 Elizabeth Ave. \\ St. John's, Newfoundland, Canada \\ A1C 5S7 \\ CORRESPONDING AUTHOR: David G. Behm \\ School of Human Kinetics and Recreation \\ Memorial University of Newfoundland \\ 230 Elizabeth Ave. \\ St. John's, Newfoundland, Canada, A1C 5S7 \\ dbehm@mun.ca \\ tel: 709-864-3408 \\ fax: 709-864-3979
}

RUNNING TITLE: Expectancy stretching effects 


\begin{abstract}
Much of the static stretching (SS) literature reports performance impairments with prolonged SS. However, it has been acknowledged that a limitation of these studies is participants' knowledge or bias. Since many participants have knowledge of the literature, their performance may be subconsciously influenced by expectations. Hence, the objective of this study was to examine the effect of stretching knowledge or deception on subsequent force output following SS. Two groups of male participants who were either aware (BIASED: 14) or unaware (DECEPTION: 14) of the SS literature participated. Unaware participants were misinformed that SS increases force production. Testing involved maximal voluntary isometric contractions (MVIC) of the quadriceps and hamstrings pre-, post-, and 5 min post-intervention (three 30s passive hamstring stretches to the point of discomfort with 30 s rest intervals) or control. While the DECEPTION group displayed impaired knee flexion force $(\mathrm{p}=0.04: 3.6 \%$ and $10.4 \%)$ following hamstrings SS, there was no significant impairment with the BIASED $(-1.1 \%$ and $+0.9 \%$ ) group. Both groups exhibited hamstrings F200 (force produced in the first $200 \mathrm{~ms}$ ) impairments following SS. Whereas BIASED participants exhibited an overall decrease $(\mathrm{p}<0.05: 1.8 \%$ and $4.2 \%)$ in knee extension MVIC, DECEPTION participants showed ( $p=0.005: 8.8 \%$ and 5.1\%) force increases. The quadriceps F200 was not significantly affected with the BIASED group but overall there were $4.5 \%$ and $8.7 \%$ F200 impairments at 1 and 5 min post-intervention $(\mathrm{p}=0.05)$ with the DECEPTION group. Thus while deception resulted in enhanced quadriceps muscle force output, there was no knowledge or deception advantage when stretching the hamstrings.
\end{abstract}

KEY WORDS: placebo, force, hamstrings, rate of force development, 


\section{INTRODUCTION}

Over the last two decades, the accumulation of stretching literature has been extensive. Two comprehensive reviews conducted by Behm and Chaouachi (2011) and Kay and Blazevich (2012) reported that prolonged ( $>60-90$ s per muscle group) static stretching (SS) impaired subsequent performance on average by 5\%-7.5\%. These SSinduced deficits may be partially related to reductions in neuromuscular activation as evidenced by decreases in electromyographic (EMG) activity (Avela et al. 1999; Behm et al. 2001; Power et al. 2004). Behm and Chaouachi (2011) indicated that a more compliant musculotendinous unit could affect the length-tension relationship as well as prolong the initial transmission of force, thus increasing rate of force development. However, a more recent position stand / review for the Canadian Society for Exercise Physiology (CSEP)(Behm et al. 2016a) illustrated that the reported SS-induced impairments are not consistent as their SS data revealed 119 significant performance reductions, 145 non-significant findings and 6 significant improvements following SS. Furthermore, the mean SS-induced performance decrement of 3.7\% was relatively lower than the prior reviews.

They also identified a number of issues that may have biased these prior results. Some of these factors included ecologically invalid stretching durations, the lack of an adequate pre-stretch warm-up or the lack of an inclusion of specific dynamic activities after the SS (Behm et al. 2016a). Furthermore, the removal of SS from the warm-up routine (Young and Behm 2002; Power et al. 2004; Shrier 2004; Magnusson and Renstrom 2006; Behm and Chaouachi 2011; Kay and Blazevich 2012) due to these reported impairments has been widely advocated and disseminated to athletes, sport 
enthusiasts and sport science / kinesiology / physical education students. As this type of research is typically pursued by sport science and kinesiology researchers, many of the participants are likely to be recruited from the same department or faculty student population who have previously been presented this stretching information. Thus, the expectation of performance impairments when SS is performed could influence study outcomes (Rosenzweig et al. 1993).

Study outcomes can be skewed with the participant's expectation of a result (Rosenzweig et al. 1993). The placebo effect illustrates that outcomes can be modified based on the expectations of individuals performing a task or treatment (Hrobjartsson et al. 2011). In a review conducted by Beedie and Foad (2009), 11 of 12 studies that implemented a placebo showed clinical significant changes in athletic performance relative to baseline. Performance placebo effects ranged from - $1.9 \%$ to $15.7 \%$ with the majority of effects between $1 \%$ and $5 \%$. As previously mentioned, two reviews reported average SS-induced impairments ranging from 3.7 - 5.0\% (Behm and Chaouachi 2011; Behm et al. 2016a). Another possibility is that researcher expectancy may introduce bias (Rosenthal 1963a; 1963b; Rosenthal et al. 1963). Thus, to reduce the likelihood of both types of bias, the CSEP position stand / review (Behm et al. 2016a) recommended that researchers should use naïve participants and researchers who are blind to treatment conditions. However there are no stretching studies that have fully implemented such an experimental protocol.

Therefore the objective of the present study was to implement a prolonged SS protocol intervention and assess the effects of using a population of participants knowledgeable of the SS literature (BIASED) versus a misinformed group lacking SS 
knowledge (DECEPTION) on subsequent agonist (stretched hamstrings) and antagonist (quadriceps) muscle force output and electromyographic (EMG) activity. It was hypothesized that the deceived group with a lack of knowledge of SS literature would minimize subsequent impairments.

\section{METHODS}

\section{Participants}

Based on prior SS studies (review: (Behm et al. 2016a)), a statistical power analysis revealed that between 8-12 subjects per group were needed to achieve an alpha of 0.05 and a power of 0.8 . Hence, 28 male students, 18 to 25 years of age were recruited. Participants' were divided into BIASED or DECEPTION groups based on their answers to SS and exercise science-related questions in a questionnaire. BIASED participants were familiar with the SS literature and the potential impairments in subsequent force production. The DECEPTION group was not cognizant of the SS literature and was falsely informed (deceived) that SS should increase subsequent muscle force output. Upon completion of the study, the DECEPTION participant group was informed of the general findings of the existing SS literature. There were no significant anthropometric or activity level differences between the groups. Both groups were considered at least recreationally active (2-3 physical activities per week). The BIASED group averaged $21.8 \pm 1.3 \mathrm{yr}, 81.6 \pm 9.9 \mathrm{~kg}$, and $179.9 \pm 7.6 \mathrm{~cm}$. The DECEPTION group averaged 21.4 \pm 2.0 yrs., $79.4 \pm 9.3 \mathrm{~kg}$, and $175.9 \pm 5.9 \mathrm{~cm}$. All participants were informed of the procedures and provided both verbal and written consent. The university Health Research Ethics Authority approved the study (HREA \#15.279).

Experimental Protocol 
The BIASED and DECEPTION groups experienced control and stretching conditions on separate days with pre- and post-intervention tests of knee flexion and extension isometric maximal voluntary contraction (MVC) forces and force produced in the first $200 \mathrm{~ms}$ (F200) as well as electromyography (EMG) of the biceps femoris and rectus femoris.

Participants attended three sessions of approximately 30 minutes duration each. During a familiarization session, the participants were informed of the protocol, required to sign an informed consent form and completed a questionnaire. The questionnaire consisted of six multiple-choice questions including one question to assess participant's knowledge of the effects of SS on force production. The remaining exercise science questions were used to ensure there was no speculation from participants as to the true research question being examined. Upon completion of the questionnaire, participants were placed in the BIASED or DECEPTION group based on their responses (knowledge or lack of knowledge regarding SS-induced impairments in the literature). Those participants unfamiliar with the SS literature were assigned to the DECEPTION group and subsequently deceived. Deception consisted of a brief verbal education session where participants were informed that due to muscle reflex activity, muscle force production increases following SS. Both groups were familiarized with the MVC and SS protocols for the subsequent sessions. The control and experimental sessions were subsequently randomized.

\section{Static Stretching Intervention}

Within 2 minutes of completing the pre-test baseline measures, participants were passively stretched using a supine hip flexion (hamstrings) static stretch. Participants 
were stretched by the same researcher (to maintain consistency) to the point of discomfort as reported by the subject. Once the point of discomfort was attained, the stretch was held for 30s. Three stretching repetitions with 30 s rest intervals were completed for each participant (Behm et al. 2004; 2011; Behm and Kibele 2007). Within 1 minute, post-intervention testing began.

\section{Testing Measures}

Testing was conducted pre-intervention, as well as 1 and 5 min post-intervention. Dependent variables included knee extension and flexion MVC forces, F200 and EMG of the rectus femoris and biceps femoris. For knee extension and flexion MVC and F200 measures, two cuffs attached to strain gauges (LCCA 500 pounds; sensitivity $=3 \mathrm{mV} / \mathrm{V}$, Omega Engineering Inc., LCCA 250, Don Mills, Ontario, Canada) via non-extensible straps were placed around participant's right ankle. Hip and knee joint angles were $90^{\circ}$. Arms were crossed at the chest. The set-up allowed measurements of knee flexor and extensor force output during a $4 \mathrm{~s}$ knee extension MVC, immediately followed by a $4 \mathrm{~s}$ knee flexion MVC. Two repetitions of each contraction pair were performed with a 4minute rest period between paired sets of contractions. The two muscle groups were tested to determine if there were differential effects of participant's SS knowledge or expectations on stretched versus non-stretched muscle groups. No physical or verbal encouragement was provided during the MVC's to reduce possible researcher bias. The contraction with the highest force output was used for analysis. All force data was sampled at a rate of $2000 \mathrm{~Hz}$ using a Biopac data acquisition system (Biopac Systems Inc. DA 100, Holliston, MA). Data were recorded and analyzed with a commercially designed software program (Acq-Knowledge III, Biopac Systems Inc.). Measures included peak 
force and F200 (peak force produced in the first $200 \mathrm{~ms}$ of the 4s MVC). According to an analytical review by Maffiuletti et al. (2016), rate of force development (RFD) measures are a stronger indication of neuromuscular responses than peak force. Although F200 is not a true measure of RFD (N vs. N.s ${ }^{-1}$ ), and has been termed instantaneous strength, it is indicative of the ability to rapidly produce force (Behm et al. 2016b).

Rectus femoris and biceps femoris EMG activity was also collected. In accordance with previously published reports from this laboratory (Behm et al. 2011; 2016b) and SENIAM recommendations (Hermens 1999). EMG electrodes were placed collar to collar $(2 \mathrm{~cm})$ on the mid-bellies of the rectus femoris and biceps femoris muscles. The rectus femoris electrodes were placed mid-way between the anterior superior iliac spine and the proximal portion of the patellar tendon. The biceps femoris electrode was placed midway between the gluteal fold and the popliteal fossa. A ground electrode was placed on the head of the fibula. Skin preparation for the electrodes included shaving, light abrading, followed by alcohol swabbing. EMG was collected using a Biopac (Biopac Systems Inc., Holliston MA) data acquisition system at a sample rate of $2000 \mathrm{~Hz}$ (amplification: 1000, impedance $=2 \mathrm{M} \Omega$, common mode rejection ratio $>$ $110 \mathrm{~dB} \min (50 / 60 \mathrm{~Hz})$, noise $>5 \mu \mathrm{V})$. A bandpass filter $(10-500 \mathrm{~Hz})$ was applied prior to digital conversion. Using a window width of 50ms, the average root mean square (RMS) EMG was calculated from the MVC over a one second period $(0.5 \mathrm{~s}$ prior and following the peak force). The mean amplitude of the RMS EMG was calculated, and used for analysis.

Statistical Analysis 
The researcher was blinded to the group and conditions when analyzing the data to avoid researcher bias. Statistical analyses were computed using SPSS software (Version 16.0, SPSS, Inc, Chicago, IL, USA). Assumption of normality (Shapiro-Wilk test) and sphericity (Mauchley test) were tested for all of the dependent variables. If the assumption of sphericity was violated, the corrected value for non-sphericity with Greenhouse-Geisser epsilon was reported. First, intraclass correlation coefficients (ICC) were measured for mean force, and EMG for the pre-tests of both conditions to assess consistency of this data. Separate repeated measures two way ANOVAs $(2 \times 3)$ were conducted for each group. Factors included two conditions (controlled and experimental) and three testing times (pre-test, 1 min post-test, and 5-minute post-test). A Bonferroni post-hoc analysis was used to detect significant differences for main effects and interactions. Cohen's d (1988) was calculated as a measure of effect size (Cohen's $d=$ $\left.\left(M_{2}-M_{1}\right) / S D_{\text {pooled }}\right)$ and the results were interpreted based on the following criteria: $<0.2$ trivial effect; $0.2-0.49$ small effect; $0.50-0.8$ moderate effect; and $>0.8$ large effect.

Descriptive statistics include means and standard deviations as well as effect size. Significance was established at 0.05 . Data was reported as means $\pm \mathrm{SD}$.

\section{RESULTS}

Reliability

ICC reliability measures for quadriceps' MVC (0.92), F200 (0.90) and EMG (0.67) exceeded hamstrings' coefficients for MVC (0.73), F200 (0.76) and EMG (0.63). Hamstrings MVC Force, F200 and EMG

There were no main effects for conditions (stretch vs. control) for any measure. There were no main effects or interactions for hamstrings EMG activity (Table 1). 
However, there were significant main effects for time within the groups with the DECEPTION MVC force and F200 as well as the BIASED F200 measures. The DECEPTION group exhibited trivial to small magnitude $3.6 \%(p=0.4$ : ES: 0.1$)$ and $10.4 \%(\mathrm{p}=0.04: \mathrm{ES}=0.3)$ decreases in hamstrings' MVC force at 1 and 5 min post-test respectively (Figure 1). Similarly, the DECEPTION group experienced trivial to small magnitude $7.0 \%(p=0.06$ : ES: 0.15$)$ and $12.2 \%(p=0.03: E S=0.27)$ decreases in hamstrings MVC F200 at 1 and 5 min post-test respectively (Figure 2). While the BIASED group did not show decrements in MVC force (Figure 1), there were small magnitude $19.6 \%(p=0.01$ : ES: 0.34$)$ and $14.9 \%(p=0.04: E S=0.22)$ decreases in hamstrings MVC F200 at 1 and 5 min post-test respectively (Figure 2). Quadriceps MVC Force, F200 and EMG

There were no main effects for conditions for any measure. There were no main effects or interactions for quadriceps EMG activity (Table 1). There was a condition $\mathrm{x}$ time interaction for the DECEPTION group quadriceps' MVC force. DECEPTION MVC force increased $8.8 \%(p=0.005: E S=0.44)$ and $5.1 \%(p=0.16: E S=0.25)$ at 1 and $5 \mathrm{~min}$ post-test respectively. Conversely during the DECEPTION control condition there were non-significant, trivial magnitude $\mathrm{MVC}$ force decreases of $3.6 \%(\mathrm{p}=0.2 \mathrm{ES}=0.13)$ and $0.6 \%(\mathrm{p}=0.4: \mathrm{ES}=0.02)$ at 1 and 5 min respectively (Figure 3$)$. The DECEPTION quadriceps F200 exhibited non-significant, trivial to small magnitude increases of 4.9\% $(p=0.1: E S=0.21)$ and $4.5 \%(p=0.1: E S=0.19)$ following the SS intervention. However there were significant moderate to large magnitude F200 impairments with the DECEPTION control condition with $13.2 \%(p=0.003)$ : $E S=0.59)$ and $20.6 \%(p=0.0004$ : $\mathrm{ES}=0.93$ ) at 1 and 5 min post-test respectively (Figure 4). There was a main effect for 
time with the BIASED group demonstrating trivial magnitude $1.8 \%(\mathrm{p}=0.1: \mathrm{ES}=0.06)$ and $4.2 \%(\mathrm{p}=0.01: \mathrm{ES}=0.15) \mathrm{MVC}$ force decrements at 1 (pre-test: $687.1 \pm 190.1 \mathrm{~N}$ vs. $674.7 \pm 200.7 \mathrm{~N}$ at $1 \mathrm{~min})$ and $5 \mathrm{~min}(657.9 \pm 187.5 \mathrm{~N})$ post-test respectively. There was no significant change in BIASED quadriceps' F200 measures.

\section{DISCUSSION}

In accordance with the bulk of the SS literature, prolonged SS of the hamstrings induced either impairments or no significant change in knee flexor MVC force, F200 and hamstrings EMG activity independent of whether the subjects were knowledgeable (BIASED group) or misinformed and not knowledgeable (DECEPTION group) of the SS literature. A second major finding was that the misinformation provided to the DECEPTION group might have provoked an increase in knee extension MVC force and F200 after prolonged SS of the hamstrings, whereas the BIASED group either had decreased quadriceps force or no change in F200.

The review / position stand by Behm et al. (2016a) warned of the possibility of participant and researcher bias with SS studies. They were concerned as to whether bias could be introduced due to participant expectancy effects (Rosenzweig et al. 1993; Beedie and Foad 2009; Hrobjartsson et al. 2011). Over the last two decades, of the approximately 150 published SS studies (Behm et al. 2016a), many have reported SSinduced impairments leading to recommendations for the removal of SS from the warmup routine (Young and Behm 2002; Shrier 2004; Magnusson and Renstrom 2006). As this information has been disseminated to researchers (Rosenthal effect: (Rosenthal 1963a; 1963b; Rosenthal et al. 1963)) and university sports/exercise science students, who form the majority of study participants, the expectation of a performance reduction 
with SS could influence study outcomes (Rosenzweig et al. 1993; Beedie and Foad 2009; Hrobjartsson et al. 2011). Thus Behm et al. (2016a) suggested that to reduce the likelihood of expectancy influences, that researchers should use naïve participants and research assistants to conduct their study or have assessors who are blind to treatment condition measure performance outcomes. In the present study, naïve participants with researchers blinded during data analysis provided mixed results.

In the present study, knowledge of the SS literature did not induce expectancy effects as both the BIASED and DECEPTION groups exhibited knee flexion F200 deficits following prolonged hamstrings SS. Also contrary to the misinformation (SS would increase force output) provided to the DECEPTION group, they experienced knee flexion MVC force deficits. Beedie and Foad in their review (2009) suggested that psychological variables such as motivation, expectancy and conditioning, and the interaction of these variables with physiological variables, could influence both positive and negative outcomes. In the present study, the adverse physiological effects of prolonged SS were more pervasive than the possible positive expectancy effects. Furthermore, although the BIASED group (primarily kinesiology undergraduate students) was knowledgeable of the SS literature and may have expected SS-induced impairments, there was no significant change in their knee flexion MVC forces. Hence, in this study, physiological responses were more prevalent than possible participant bias or expectancy effects when examining the stretched muscle group. However, there may have been some evidence of expectancy effects with the antagonist (quadriceps) muscle group.

It is possible that the misinformation provided to the DECEPTION group inspired an augmentation of knee extension MVC force and F200 after prolonged hamstrings SS. 
It is also conceivable that prolonged hamstrings SS decreased the passive musculoskeletal stiffness or active co-contractile activity resulting in less antagonist resistance to the knee extension MVC (Behm et al. 2016a). However, the BIASED group's significant knee extension MVC force deficits and lack of change with F200 would argue against changes in hamstrings co-activation augmenting quadriceps MVC force and F200.

On the other hand, stretching the hamstrings might have positive effects on quadriceps performance via reflex potentiation mechanisms. However, studies examining the non-local effects of stretching have reported either no effect (Chaouachi et al. 2016; Behm et al. 2016b) or impairments (Lima et al. 2014; Marchetti et al. 2014) in the performance of a non-stretched muscle group. For example, unilateral stretching of the quadriceps increased contralateral hip extension range of motion (ROM) but did not affect isokinetic torque (Chaouachi et al. 2016). Stretching either the upper or lower body enhanced ROM in the lower or upper body respectively but did not impair isometric force (Behm et al. 2016b). However, stretching the upper body (Marchetti et al. 2014) or unilateral plantar flexors (Lima et al. 2014) adversely affected jump height. Since the BIASED and DECEPTION groups displayed differing quadriceps responses to prior hamstrings SS and the scant literature on non-local muscle responses to SS show either no effect or deficits, it is unlikely the DECEPTION group increases in quadriceps MVC force or F200 can be attributed to a physiological response to prolonged hamstrings SS. The DECEPTION group was informed that SS would generally improve force output, not specifically the force output of only the stretched muscle group. Hence, it is possible that the misinformation provided to the DECEPTION group had a positive effect on 
quadriceps performance, since there were no counteracting inhibitory physiological influences (i.e. prolonged SS of the quadriceps).

Since knee flexion and extension contractions were performed consecutively, it could be argued that reciprocal inhibition might have contributed to decreased knee flexion force. However, this is unlikely as 1) there are a vast number of SS studies that only used single contractions of the stretched muscle and reported deficits, 2) if reflex inhibition was substantial, it should work in both directions (hamstrings $\leftrightarrow$ quadriceps) but there was no knee extension force impairments present and in fact potentiation occurred in the DECEPTION group and 3) there was no change in EMG activity. The lack of significant change with hamstrings and quadriceps EMG activity in light of force changes might be initially perplexing. However, since the EMG-force relationship is not linear but actually curvilinear with an EMG plateau at higher force levels (BiglandRitchie 1981), small MVC force changes would not be reflected by similar changes in the EMG activity.

\section{CONCLUSIONS}

Whereas the physiological impairments associated with prolonged hamstrings SS possibly overcame positive psychological expectancy effects with the knee flexion MVC force production with the DECEPTION group; the lack of prior SS of the quadriceps may have permitted the positive expectancy effects to emerge. Furthermore, the biased group (with knowledge that the literature indicates SS tends to lead to subsequent impairments) actually did not experience knee flexion MVC force deficits. Participant bias or deception effects in the present findings were generally of trivial to small magnitudes and thus the findings in the SS literature should not be strongly affected by prior knowledge. 
However, there is some evidence that expectancy effects could have a small influence (i.e. non-stretched knee extension forces) on performance outcomes.

CONFLICT OF INTEREST: All authors declare no conflicts of interest with this publication.

\section{ACKNOWLEDGMENTS}

Authors would like to acknowledge the contribution of Dr. Thamir Alkanani. The authors declare no funding sources, or conflict of interest with this study. Authors declare that the results of the study are presented clearly, honestly, and without fabrication, falsification, or inappropriate data manipulation.

\section{REFERENCES}

Avela, J., KyrîlÑinen, H., Komi, P.V. 1999. Altered reflex sensitivity after repeated and prolonged passive muscle stretching. J. Appl. Physiol. 86(4): 1283-1291.

Beedie, C.J. and Foad, A.J. 2009. The placebo effect in sports performance: a brief review. Sports Med. 39: 313-29.

Behm, D.G., Bambury, A., Cahill, F., and Power, K. 2004. Effect of acute static stretching on force, balance, reaction time, and movement time. Med. Sci. Sports Exerc. 36: $1397-402$.

Behm, D.G., Blazevich, A.J., Kay, A.D., and McHugh, M. 2016a. Acute effects of muscle stretching on physical performance, range of motion, and injury incidence in healthy active individuals: a systematic review. Appl. Physiol. Nutr. Metab. 41: 1-11. Behm, D.G., Button, D.C., Butt, J.C. 2001. Factors affecting force loss with prolonged stretching. Can. J. Appl. Physiol. 26(3): 261-272.

Behm, D.G., Cavanaugh, T., Quigley, P., Reid, J.C., Nardi, P.S., and Marchetti, P.H. 2016b. Acute bouts of upper and lower body static and dynamic stretching increase nonlocal joint range of motion. Eur. J. Appl. Physiol. 116: 241-9.

Behm, D.G. and Chaouachi, A. 2011. A review of the acute effects of static and dynamic stretching on performance. Eur. J. Appl. Physiol. 111: 2633-51.

Behm, D.G. and Kibele, A. 2007. Effects of differing intensities of static stretching on jump performance. Eur. J. Appl. Physiol. 101: 587-94.

Behm, D.G., Plewe, S., Grage, P., Rabbani, A., Beigi, H.T., Byrne, J.M., and Button, D.C. 2011. Relative static stretch-induced impairments and dynamic stretch-induced enhancements are similar in young and middle-aged men. Appl. Physiol. Nutr. Metab. 36: 790-7.

Bigland-Ritchie, B. 1981. EMG/force relations and fatigue of human voluntary contractions. Exerc. Sport Sci. Reviews 9: 75-111. 
Chaouachi, A., Padulo, J., Kasmi, S., Othmen, A.B., Chatra, M., and Behm, D.G. 2016. Unilateral static and dynamic hamstrings stretching increases contralateral hip flexion range of motion. Clin. Physiol. Funct. Imaging. doi: 10.1111/cpf.12263

Cohen J. 1988. Statistical power analysis for the behavioural sciences. L. Erbaum Associates, Hillside, pp 14-68

Hermens, H.J.M., Merlatti, R.; Freriks, B. 1999. European recommendations for surface electromyography results of the SENIAM project. Enschede, Netherlands: Roessingh Research and Development. pp. 12-86

Hrobjartsson, A., Kaptchuk, T.J., and Miller, F.G. 2011. Placebo effect studies are susceptible to response bias and to other types of biases. J. Clin. Epidemiol. 64: 1223-9. Kay, A.D. and Blazevich, A.J. 2012. Effect of acute static stretch on maximal muscle performance: a systematic review. Med. Sci. Sports Exerc. 44: 154-64.

Lima, B.N., Lucareli, P.R., Gomes, W.A., Silva, J.J., Bley, A.S., Hartigan, E.H., and Marchetti, P.H. 2014. The acute effects of unilateral ankle plantar flexors staticstretching on postural sway and gastrocnemius muscle activity during single-leg balance tasks. J. Sports Sci. Med. 13: 564-70.

Maffiuletti, N.A., Aagaard, P., Blazevich, A.J., Folland, J., Tillin, N., and Duchateau, J. 2016. Rate of force development: physiological and methodological considerations. Eur. J. Appl. Physiol. DOI: 10.1007/s00421-016-3346-6

Magnusson, P. and Renstrom, P. 2006. The European College of Sports Sciences Position statement: The role of stretching exercises in sports. Eur. J. Sport Sci. 6: 87-91.

Marchetti, P.H., Silva, F.H., Soares, E.G., Serpa, E.P., Nardi, P.S., Vilela, B., and Behm, D.G. 2014. Upper limb static-stretching protocol decreases maximal concentric jump performance. J. Sports Sci. Med. 13: 945-50.

Power, K., Behm, D., Cahill, F., Carroll, M., and Young, W. 2004. An acute bout of static stretching: effects on force and jumping performance. Med. Sci. Sports Exerc. 36: 1389-96.

Rosenthal, R. 1963a. Experimenter attributes as determinants of subjects' responses. J. Proj. Tech. 27: 324-31.

Rosenthal, R. 1963b. Experimenter Modeling Effects as Determinants of Subject's Responses. J. Proj. Tech. Pers. Assess. 27: 467-71.

Rosenthal, R., Persinger, G.W., Kline, L.V., and Mulry, R.C. 1963. The Role of the Research Assistant in the Mediation of Experimenter Bias. J. Pers. 31: 313-35.

Rosenzweig, P., Brohier, S., and Zipfel, A. 1993. The placebo effect in healthy volunteers: influence of experimental conditions on the adverse events profile during phase I studies. Clin. Pharmacol. Ther. 54: 578-83.

Shrier, I. 2004. Does stretching improve performance?: a systematic and critical review of the literature 39. Clin. J. Sport Med. 14: 267-273.

Young, W. and Behm, D. 2002. Should static stretching be used during a warm-up for strength and power activities? Strength Cond. J. 24: 33-37. 
Table 1: Electromyographic (EMG) activity (mV). Mean \pm standard deviation

\begin{tabular}{|l|l|l|l|l|}
\hline & \multicolumn{2}{|c|}{ BIASED GROUP } & \multicolumn{2}{c|}{ DECEPTION GROUP } \\
\hline & $\begin{array}{l}\text { Stretch } \\
\text { Intervention }\end{array}$ & Control & $\begin{array}{l}\text { Stretch } \\
\text { Intervention }\end{array}$ & Control \\
\hline Pre-test & $0.190 \pm 0.056$ & $0.150 \pm 0.045$ & $0.169 \pm 0.058$ & $0.170 \pm 0.068$ \\
\hline Post-test & $0.186 \pm 0.062$ & $0.152 \pm 0.054$ & $0.165 \pm 0.058$ & $0.163 \pm 0.067$ \\
\hline 5 min post-test & $0.164 \pm 0.067$ & $0.143 \pm 0.038$ & $0.159 \pm 0.055$ & $0.159 \pm 0.067$ \\
\hline
\end{tabular}




\section{FIGURE LEGENDS}

Figure 1: Main effect for time: DECEPTION and BIASED groups MVC force. The "p" value for the DECEPTION condition illustrates differences from pre-test. Other comparisons were not statistically significant.

Figure 2: Main effect for time: DECEPTION and BIASED groups MVC F200 (force produced in the first 200ms). Both " $p$ " values for DECEPTION condition illustrate differences from pre-test.

Figure 3: DECEPTION group knee extension MVC force condition x time interaction. Both " $p$ " values illustrate differences from pre-test.

Figure 4: DECEPTION group knee extension MVC F200 (force produced in the first 200ms) condition $\mathrm{x}$ time interaction. Both "p" values illustrate differences from pre-test. 
Figure 1

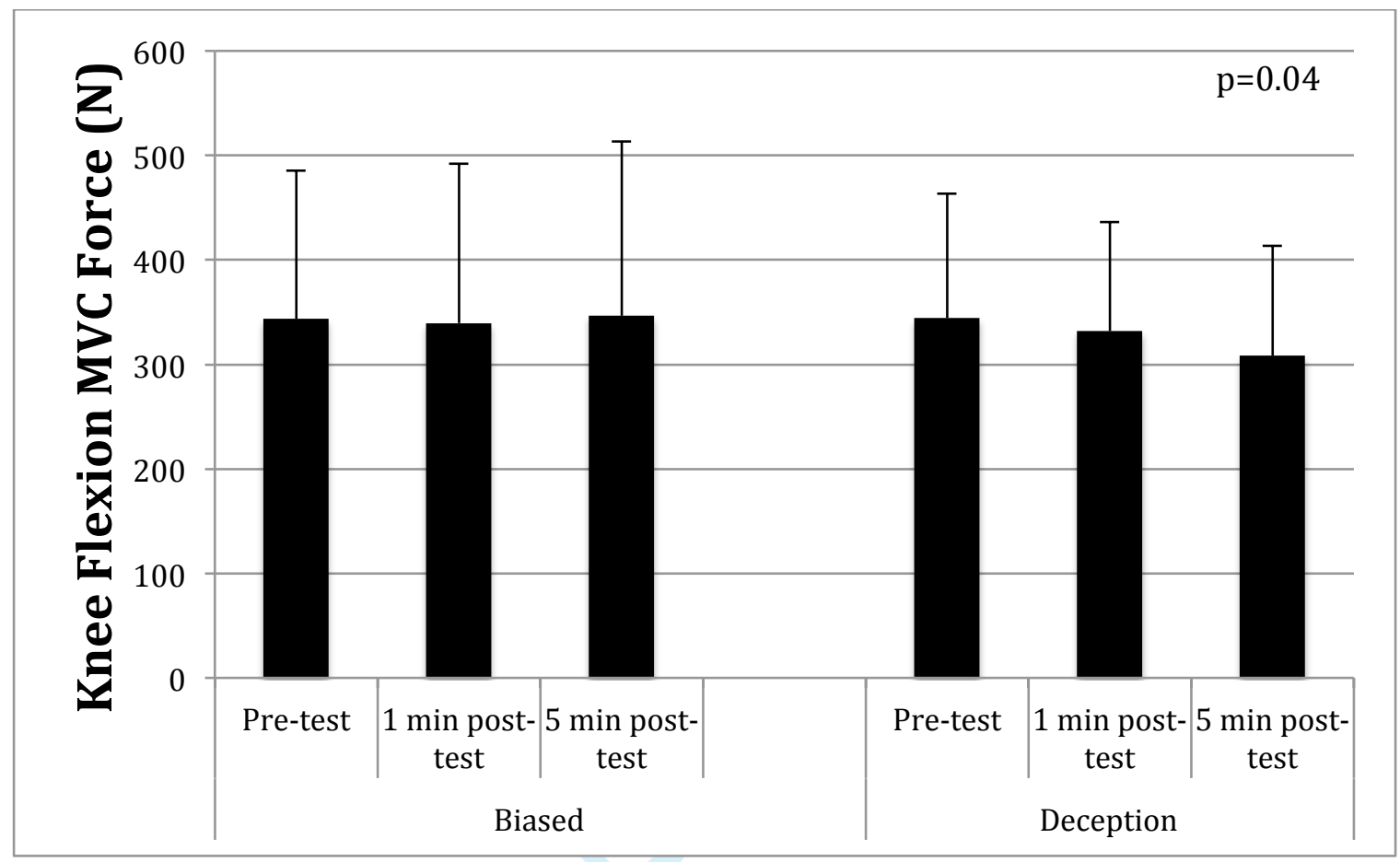


Figure 2

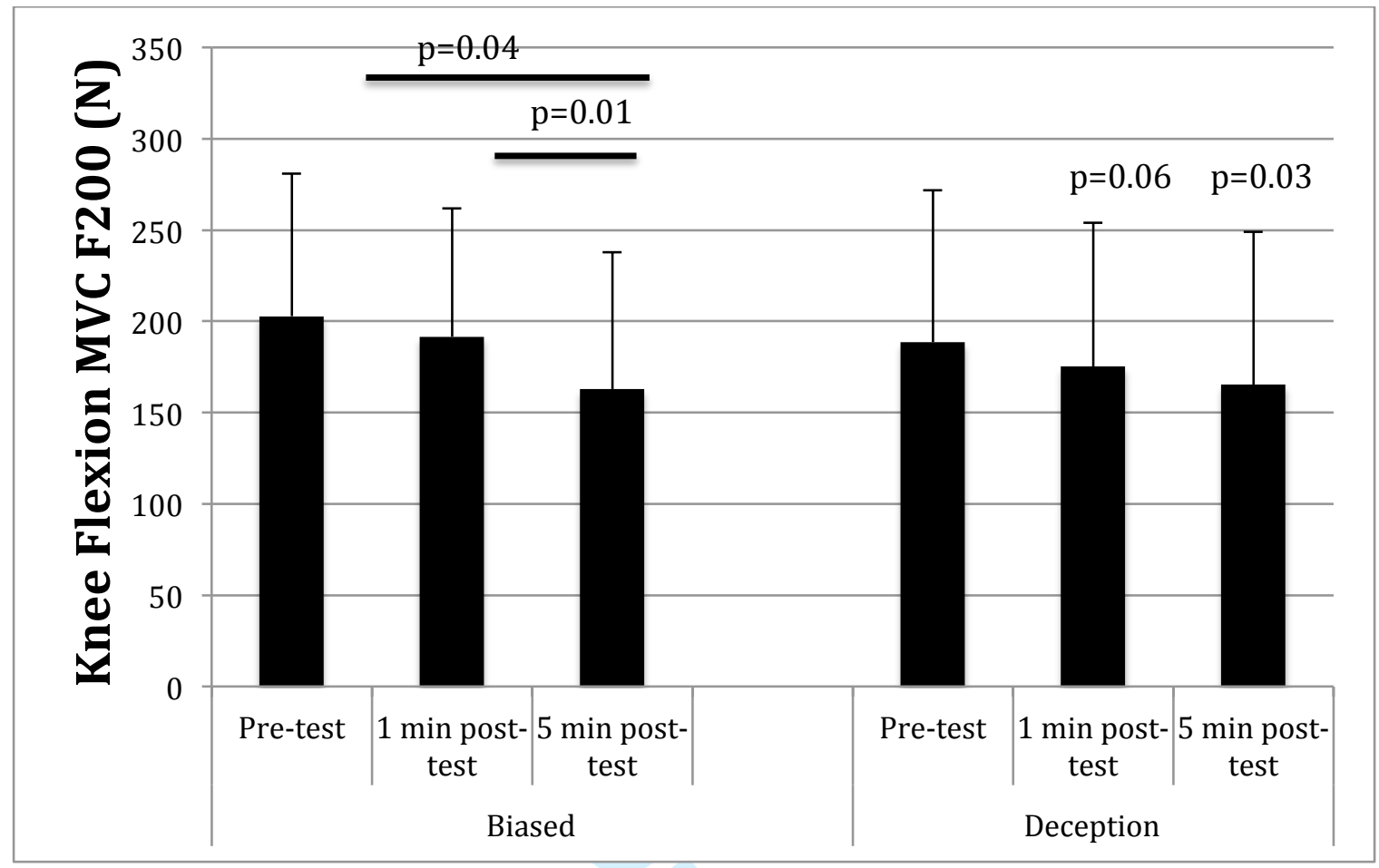


Figure 3

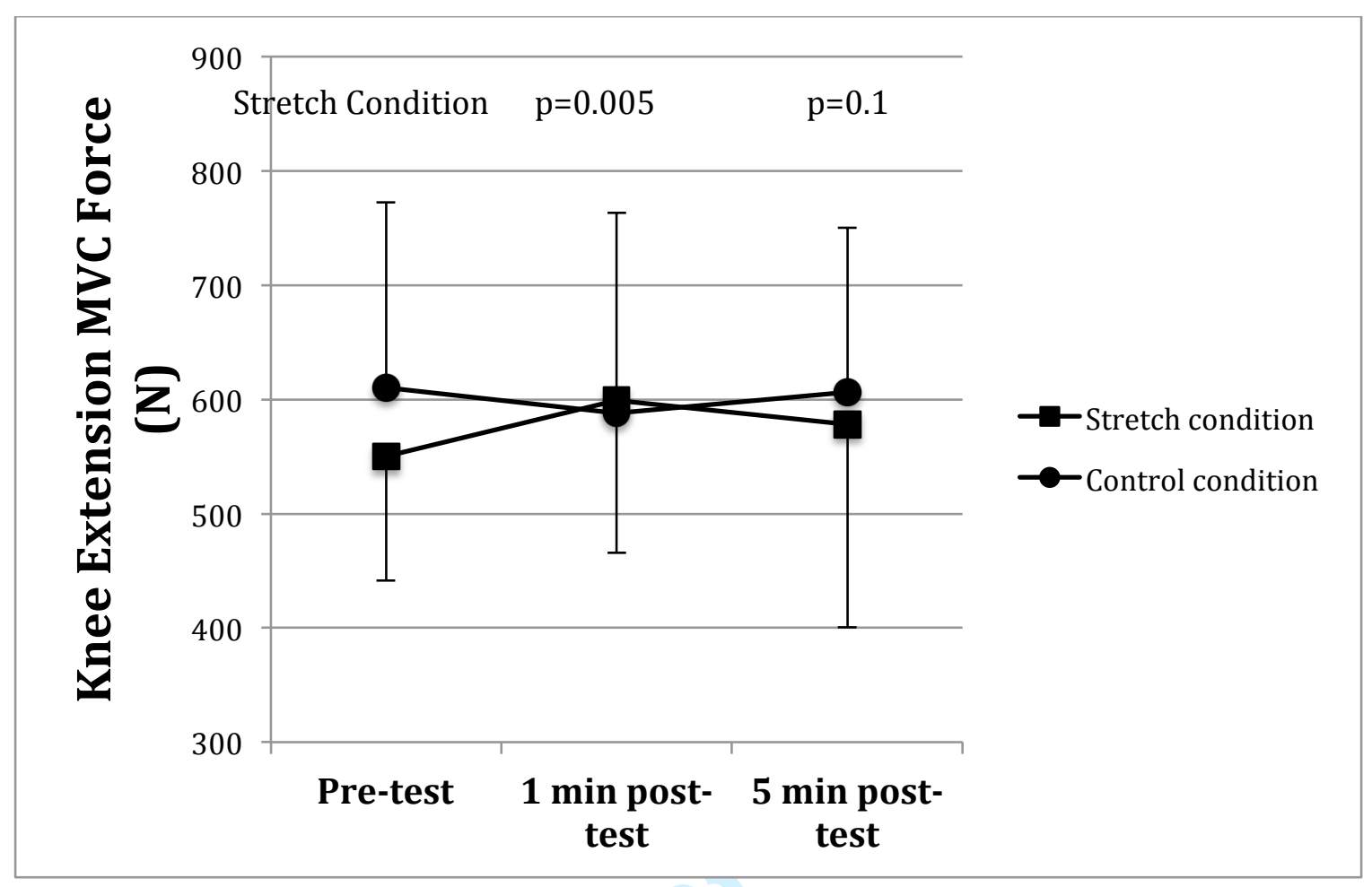


Figure 4

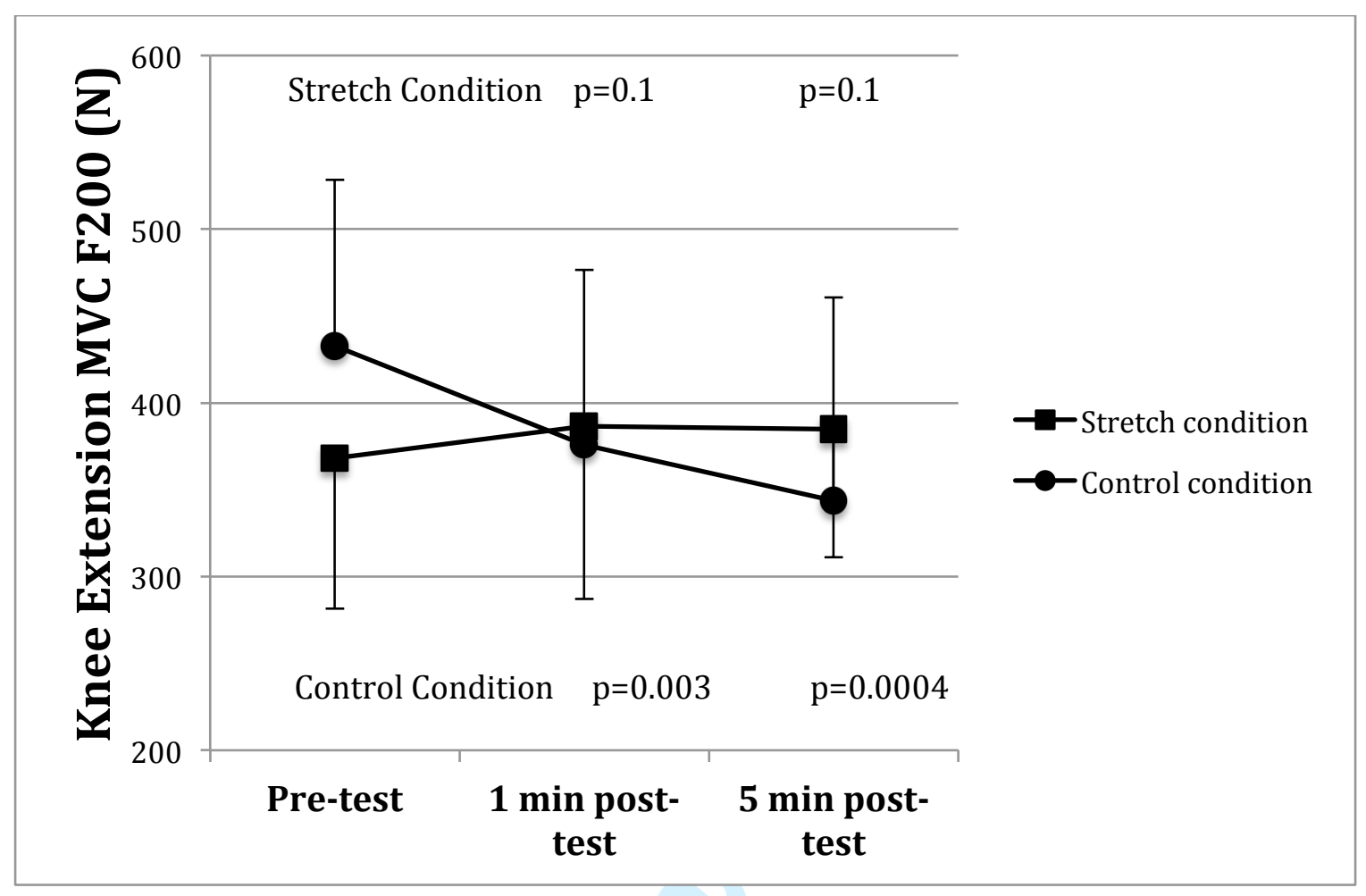

